\title{
AVALIAÇÃO DA ATIVIDADE ANTIFÚNGICA DE FOLHAS DE CINAMOMO (Melia azedarach) SOBRE FUNGOS FITOPATOGÊNICOS VISANDO UMA ALTERNATIVA AOS AGROTÓXICOS
}

\author{
Jade Ayres Barbedo Martins ${ }^{1}$ e César Luis Siqueira Junior ${ }^{2}$
}

\begin{abstract}
Resumo: Compostos bioativos de origem vegetal têm sido largamente estudados por serem fontes naturais de substâncias antifúngicas. No presente trabalho, utilizou-se um extrato hidroetanólico para extrair compostos bioativos de folhas de plantas de Melia azedarach. A atividade antifúngica do extrato de $M$. azedarach foi então testada contra três fungos fitopatogênicos: Fusarium solani, Fusarium oxysporum e Colletotrichum gloeosporioides, este último, muito conhecido por ser causador de antracnose em frutos de mamão (Carica papaya L.) no período pós-colheita. O extrato hidroetanólico exibiu forte potencial inibitório sobre o crescimento micelial do F. oxysporum reduzindo em 85 \% seu desenvolvimento. Essa inibição é corroborada pela mudança morfológica causada nos micélios fúngicos pela adição de 100 ppm do extrato. Foi observada ainda, uma redução de $44 \%$ do crescimento do F. solani, na presença do extrato. Apesar da leve inibição provocada pelo extrato no desenvolvimento do fungo C. gloeosporioides (14\%), quando aplicado nos frutos, o extrato confere a redução do desenvolvimento de sintomas da antracnose mantendo a qualidade visual dos frutos testados. Os resultados obtidos nesse trabalho corroboram a ideia de uso de compostos extraídos de plantas como uma alternativa sustentável à aplicação de agrotóxicos promovendo o barateamento do processo de controle de pragas e patógenos no cultivo, além de constituir uma forma de controle menos agressiva ao ambiente.
\end{abstract}

Palavras-chaves: Antracnose. Extrato vegetal. Atividade antifúngica. Mamão.

\section{Introdução}

A grande demanda por produtos de boa aparência e qualidade, pelos consumidores, tem exigido atenção dos produtores que, por sua vez, precisam adequar os cuidados fitossanitários da produção assim como do período pós-colheita dos frutos. Boa parte das perdas de frutos nessa etapa da produção ocorre pela ação de fungos, que são os principais responsáveis pelo aparecimento de doenças que resultam na perda de qualidade dos frutos no póscolheita (VENTURA; COSTA, 2003).

Fungicidas químicos têm sido largamente empregados como forma principal de controle das doenças do pós-colheita provocadas por fungos, como, por exemplo, a antracnose causada por fungos do gênero Colletotrichum sp. em várias plantas. $\mathrm{Na}$ cultura de mamão, por exemplo, a incidência da antracnose, causada pelo fungo $C$. gloeosporioides é controlada pela aplicação de uma gama de fungicidas. Dentre esses fungicidas, destacam-se 0 tebuconazol (triazol), tiabendazol (benzimidazol) e mancozeb (ditiocarbamato) (AGROFIT, 2020), todos considerados muito perigosos ao meio ambiente. $\mathrm{O}$ uso desses fungicidas em frutos colhidos tem sido largamente contestado por conta dos problemas relacionados à forte toxicidade residual que acaba causando danos ao ambiente e ao desenvolvimento de pragas resistentes, além de causarem danos sérios a saúde humana pela exposição direta prolongada aos compostos (LUNDSTRÖM et al., 2017). Estudos demonstram que alguns desses compostos, como os carbamatos são capazes de causar danos no sistema nervoso de organismos aquáticos por afetar a atividade de enzimas necessárias ao metabolismo (WANG et al., 2015). Esses compostos, apesar de serem utilizados em lavouras no controle de pragas, apresentam alto risco ecológico, pois podem levar a contaminação do ambiente aquático. Mesmo assim, somente em 2020, mais dois novos fungicidas à base de mancozeb foram liberados para as culturas de mamão (MAPA, 2020). Os efeitos indesejáveis provocados pelos fungicidas químicos tornam imperativo

$\overline{1}$ E-mail: jade_abm@yahoo.com.br 
a busca por novas alternativas para o combate as doenças do pós-colheita capazes de reduzir perdas dos frutos, mas que sejam, ao mesmo tempo, seguras ao ambiente e a saúde humana (AHMADU et al., 2020).

Extratos vegetais aparecem, então, como uma alternativa promissora para o controle de patógenos e aumento do tempo de vida dos frutos no pós-colheita permitindo a comercialização dos mesmos, sem impactos ambientais e demais danos à saúde (MOHAMED; EL-HADIDY, 2008; MARTINS; SIQUEIRA-JUNIOR, 2017). Várias espécies de plantas vêm sendo descritas por apresentarem potencial antimicrobiano, e essa propriedade se deve ao fato de as plantas produzirem substâncias naturais que são tóxicas a alguns fungos causadores de doenças em plantas (ADEME et al., 2013; NAZ; BANO, 2013; ROSADO-ÁLVAREZ et al., 2014; AHMADU et al., 2020). Nesse contexto, o presente trabalho teve como objetivo determinar o potencial antifúngico de extrato foliar de $M$. azedarach sobre os fungos F. solani, F. oxysporum e C. gloeosporioides, causadores de doenças em plantas de mamoeiro.

\section{Metodologia}

O presente trabalho foi desenvolvido no Laboratório de Bioquímica e Função de Proteínas Vegetais, pertencente ao Departamento de Botânica, da Universidade Federal do Estado do Rio de Janeiro.

Folhas de cinamomo (Melia azedarach) foram coletadas no período de março de 2012 a julho de 2014, sempre no início da manhã, no município de Campos dos Goytacazes/RJ (-21.748863, -41.169285). A planta foi identificada pela Profa. Laura Jane Moreira Santiago, da Universidade Federal do Estado do Rio de Janeiro, e uma amostra foi depositada no herbário HUNI, gerando um número de Voucher (HUNI 1374). As amostras foliares foram secas a $40^{\circ} \mathrm{C}$ até a observação de um peso constante. As folhas desidratadas foram pulverizadas em moinho de facas e estocadas em recipientes hermeticamente fechados para posterior uso.

Os microrganismos testados nesse trabalho, Fusarium solani e Fusarium oxysporum foram obtidos a partir da micoteca do Laboratório de Fisiologia e Bioquímica de Microrganismos da Universidade Estadual do Norte Fluminense - Darcy Ribeiro, Rio de Janeiro, Brasil. $\mathrm{O}$ microrganismo Colletotrichum gloeosporioides, foi isolado a partir de frutos de mamão contaminados obtidos no mercado local. Todos os microrganismos foram mantidos em meio BDA (Batata 4g/L, Dextrose $20 \mathrm{~g} / \mathrm{L}$, Agar 15 g/L).

Os compostos naturais de $M$. azedarach foram extraídos a partir de $100 \mathrm{~g}$ de tecido foliar pulverizado usando solução etanol:água (7:3 v/v). Após incubação por 7 dias, a mistura foi filtrada e o solvente removido por evaporação em rotavapor. O material resultante foi solubilizado em dimetilsulfoxido (DMSO). O extrato foi então esterilizado por filtração em filtro de seringa $(0,2 \mu \mathrm{m})$ e mantido ao abrigo da luz à $4^{\circ} \mathrm{C}$ até o uso.

A atividade antifúngica de folhas de erva-de-santa-barbara foi avaliada in vitro baseando-se na metodologia descrita por Siqueira Junior et al. (2012), com algumas modificações. Diferentes concentrações (10, 50, $100 \mathrm{ppm}$ ) foram misturadas em meio BDA fundente e adicionados a placas de Petri para um volume total de $20 \mathrm{~mL} /$ placa. Após solidificado, um disco micelial de $5 \mathrm{~mm}$ de cada espécie de fungo testada foi inoculado no centro de cada placa. As placas foram mantidas a $25^{\circ} \mathrm{C}$ com fotoperíodo de $12 \mathrm{~h}$ claro/12 h escuro por um período de 7 dias. Foram tomadas as medidas radiais $(\mathrm{mm})$ das colônias em cada placa e a inibição fúngica foi calculada com referência à placa controle, contendo apenas fungo inoculado em meio BDA (na ausência de extrato).

Mudanças morfológicas na estrutura micelial dos fungos testados, causadas pela ação do extrato foram avaliadas através da metodologia descrita por Chen e Dai (2012). Resumidamente, discos miceliais (com diâmetro de $5 \mathrm{~mm}$ ) originados de culturas fúngicas com 10 dias de cultivo foram adicionados em frascos Erlenmeyer contendo $50 \mathrm{~mL}$ de meio BD (Batata, Dextrose) e diferentes concentrações de extrato de ervade-santa-barbara (10, 50, 100 ppm). O material foi então incubado a $25{ }^{\circ} \mathrm{C}$ sob agitação constante em shaker orbital (ES-20, Biosan) por 7 dias. Amostras controle continham apenas o inóculo fúngico em meio BD. Após o período de incubação, as mudanças morfológicas nos micélios de cada fungo testado foram observadas utilizando um microscópio óptico. Todos os tratamentos foram feitos em triplicata.

Antracnose, uma doença causada em frutos de mamão pelo fungo C.gloeosporioides, foi usada como sistema modelo para avaliação do potencial inibitório in vivo do extrato de $M$. azedarach. Frutos de 
mamão em estágio 4 de amadurecimento (mais amarelo que verde), foram adquiridos em mercados locais. Trinta frutos de mamão foram esterilizados com banho de etanol $70 \%$ por $5 \mathrm{~min}$ e em cada fruto foram produzidos ferimentos $(0,5 \mathrm{~cm}$ de profundidade e $0,2 \mathrm{~cm}$ de diâmetro) em três posições na superfície dos frutos. Alguns frutos (15 frutos) foram então mergulhados por $5 \mathrm{~min}$. em solução contendo 100 ppm do extrato vegetal estudado. Os outros 15 frutos foram mergulhados em solução de extração etanol:água $(7: 3 \mathrm{v} / \mathrm{v})$ na ausência de extrato (controle). Os frutos foram então armazenados para secagem natural, e em seguida, em cada ferimento produzido foi adicionado um disco micelial $(0,1 \mathrm{~cm})$ do fungo $C$. gloeosporioides. Os frutos foram acondicionados em bandejas (simulando seu armazenamento natural durante o transporte para comercialização), a $25^{\circ} \mathrm{C}$ por 7 dias. Ao final do período de incubação foi observado o aparecimento de lesões, consideradas como sintoma típico da antracnose.

O delineamento experimental utilizado foi o Delineamento Inteiramente Casualizado em esquema fatorial $3 \times 4$ (três fungos: $F$. solani, $F$. oxysporum e $C$. gloeosporioides) três concentrações do extrato vegetal $(10,50$ e $100 \mathrm{ppm})$ mais o controle $(0 \mathrm{ppm})$ com três repetições. As médias foram comparadas pelo teste de Turkey $(p<0,05)$ utilizando o programa ASSISTAT versão 7.7 beta desenvolvido pelo Professor Dr. Francisco da Universidade Federal de Campina Grande (SILVA; AZEVEDO, 2016). Para os ensaios in vivo foram utilizados 90 frutos, divididos em três repetições de 30 frutos.

\section{Resultados}

A partir dos resultados obtidos ao longo desse trabalho verificou-se que 0 extrato bruto de $M$. Azedarach exibiu uma significativa atividade antifúngica in vitro contra os fungos $F$. oxysporum e $F$. solani. $O$ crescimento micelial de ambos os fungos foi reduzido pelo tratamento com o extrato de maneira dose dependente, observando-se a redução do crescimento fúngico com o aumento das doses do extrato (Tabela 1). Os resultados sugerem que fungo $F$. oxysporum tem seu desenvolvimento severamente prejudicado pelo extrato vegetal, que provoca uma inibição de cerca de $85 \%(p<0,05)$ no crescimento micelial do fungo, que na presença do extrato atinge um diâmetro de $9,2 \mathrm{~mm}$, enquanto o da amostra controle atinge $63,11 \mathrm{~mm}$. De maneira similar, o fungo $F$. solani tem seu crescimento inibido pelo extrato vegetal que com uma concentração de $100 \mathrm{ppm}$, leva a redução de aproximadamente $50 \%$ do crescimento micelial do fungo (Tabela 1).

Plantas da espécie $M$. azedarach têm sido descritas por suas propriedades antifúngicas sobre fungos fitopatogênicos. Sen e Batra (2012) obtiveram inibição efetiva do crescimento dos fungos Aspergillus flavus e Rhizopus stolonifer com extratos de $M$. azedarach obtidos com vários solventes como etanol, metanol e éter de petróleo. Mais recentemente, Seepe et al. (2020) demonstraram a eficácia do extrato de folhas de $M$. azedarach, usando acetona como solvente, capaz de inibir $97 \%$ do crescimento do fungo Fusarium proliferatum. Esses dados corroboram os resultados obtidos neste trabalho, demonstrando o potencial antifúngico das folhas de $M$. azedarach.

Tabela 1 - Efeito do extrato de M. azedarach sobre o crescimento micelial in vitro dos fitopatógenos

\begin{tabular}{cccc}
\hline & \multicolumn{3}{c}{ Crescimento micelial $(\mathbf{m m})$} \\
\cline { 2 - 4 } [Concentração] & C. gloeosporioides & F. solani & F. oxysporum \\
\hline Controle & $63,00 \mathrm{aA}$ & $61,11 \mathrm{aA}$ & $63,11 \mathrm{aA}$ \\
$10 \mathrm{ppm}$ & $66,11 \mathrm{aA}$ & $58,77 \mathrm{aAB}$ & $52,55 \mathrm{bB}$ \\
$50 \mathrm{ppm}$ & $62,78 \mathrm{aA}$ & $46,67 \mathrm{bB}$ & $23,22 \mathrm{cC}$ \\
$100 \mathrm{ppm}$ & $54,11 \mathrm{bA}$ & $33,78 \mathrm{cB}$ & $9,20 \mathrm{dC}$ \\
\hline
\end{tabular}

Todos os dados são expressos como média $(n=36)$. Letras minúsculas diferentes em cada coluna diferem estatisticamente entre si comparadas ao grupo controle pelo teste de Turkey a $5 \%$ de probabilidade. Letras maiúsculas diferentes em cada linha diferem estatisticamente entre si comparadas aos organismos testados pelo teste de Turkey a $5 \%$ de probabilidade.

Fonte: Autores (2020)

Aparentemente, o extrato vegetal de M. azedarach não interfere significativamente no crescimento micelial in vitro fungo $C$. gloeosporioides mesmo na maior concentração utilizada nos ensaios (100 ppm) levando a uma inibição de cerca de $15 \%$ (Tabela 1). 


\section{REA - Revista de estudos ambientais (Online)}

v.22, n. 2, p.43-49, jul./dez. 2020

Alterações morfológicas no crescimento micelial dos fungos na presença do extrato de $M$. azedarach também foram avaliadas por meio do crescimento dos fungos em meio líquido contendo 100 ppm de extrato vegetal. Como resultado, foi observada a redução geral do crescimento micelial dos fungos $F$. solani e $F$. oxysporum testados. Em relação ao controle (Figura 1 $B, C)$, os micélios de ambos os fungos tratados com o extrato apresentam uma alteração morfológica com hifas mais curtas e mais ramificadas (Figura $1-E, F$ ). Além disso, observam-se micélios menores do que os dos fungos não tratados reforçando a ideia de que o extrato exerce potencial inibitório sobre o crescimento desses fungos. A leve inibição do fungo $C$. gloeosporioides, provocada pelo extrato vegetal nos tratamentos in vitro, é corroborada com a análise das alterações morfológicas provocadas no fungo pela presença do extrato vegetal em meio de cultura. É possível verificar que há uma tênue variação na forma das hifas, que mantêm o mesmo crescimento comparadas as hifas da amostra controle (Figura 1 - A, D). Contudo, são observados vários esporos na amostra controle (Figura 1 - A), o que não é visualizado na amostra tratada (Figura $1-\mathrm{D})$, indicando uma redução do número de esporos produzidos após o tratamento.

Figura 1 - Observação microscópica da influência de 100 ppm do extrato de Melia azedarach na morfologia micelial de fungos após incubação das culturas por 7 dias a $25^{\circ} \mathrm{C}$. Colletotrichum gloeosporioides (A e D), (A) controle, (D) tratado; Fusarium solani (B e E), (B) controle, (E) tratado; Fusarium oxysporum (C e F), (C) controle, (F) tratado. $O$ resultado é uma figura representativa de um dos três experimentos independentes. Todas as fotos foram produzidas com a mesma ampliação, conforme demonstrado na figura $F$ contendo a barra medindo $20 \mu \mathrm{m}$
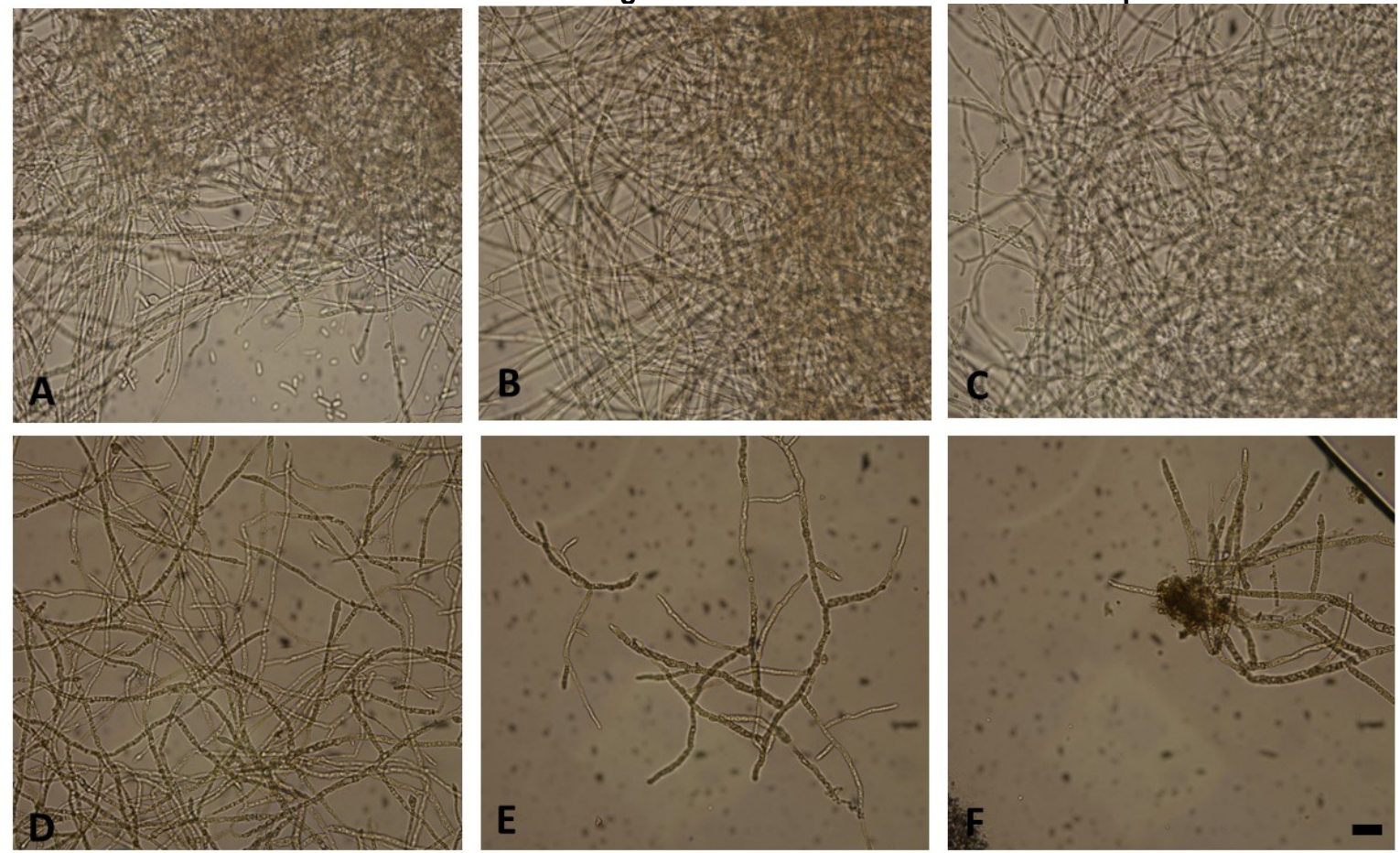

Fonte: Autores (2020)

Alterações morfológicas provocadas pelo tratamento com extratos vegetais já foram observadas em outros trabalhos. Chen e Dai (2012), por exemplo, também demonstraram que na presença de extratos de Cinnamomum camphora ocorriam mudanças morfológicas em micélios do fungo Colletotrichum lagenarium causador de antracnose em pepino. Da mesma forma, foram demonstradas modificações morfológicas, como a perda de esporulação e a despigmentação da cabeça de conídios espécies de Aspergilus quando submetidos ao extrato hidroetanólico de Lamium purpureum (GRUJIĆ et al., 2020). Essas mudanças morfológicas provocadas pelos tratamentos com os extratos vegetais podem ser importantes para o processo de inibição fúngica por estarem associadas a estruturas importantes para processos metabólicos 
envolvidos na virulência e no ciclo de vida do patógeno.

Em frutos de mamão, a antracnose, doença provocada pelo fungo $C$. gloeosporioides no período pós-colheita causa grandes perdas econômicas devido aos sintomas típicos da doença que impossibilita a comercialização dos frutos contaminados. Ainda no campo, o fungo infecta o fruto mantendo-se latente durante 0 desenvolvimento do mesmo, até o período de amadurecimento, quando então os sintomas aparecem (CAPDEVILLE et al., 2007). Apesar de in vitro, o extrato de $M$. azedarach não provocar uma forte inibição no crescimento do fungo, o tratamento in natura com o extrato provoca uma significante diferença $(p<0,05)$ nos frutos inoculados após o tratamento comparados aos frutos não tratados. A incidência de antracnose foi significativamente menor nos frutos tratados do que nos frutos controle, como pode ser observado na Figura 2. Os frutos tratados com o extrato vegetal apresentaram os sintomas reduzidos (Figura $2-B, D$ ), comparados aos sintomas típicos da antracnose bem visíveis na amostra controle (Figura 2 - A, C). A diminuição da severidade dos sintomas de antracnose nos frutos tratados poderia ser facilmente explicada pela redução da germinação de esporos, também observada nas análises por microscopia. Na presença do extrato vegetal na superfície da casca dos frutos, os esporos fúngicos não germinam, reduzindo assim a formação de hifas e consequentemente 0 aparecimento dos sintomas associados a doença.

Figura 2 - Efeito do extrato de $M$. azedarach sobre a incidência natural de antracnose em frutos infectados. Como controle, 15 frutos foram tratados com solução de extração (A); outros 15 frutos foram tratados com $100 \mathrm{ppm}$ de extrato vegetal (B). As setas indicam os sintomas de antracnose nos frutos tratados, em detalhes no fruto controle (C) e no fruto tratado (D). O resultado é uma foto representativa de um dos três experimentos independentes

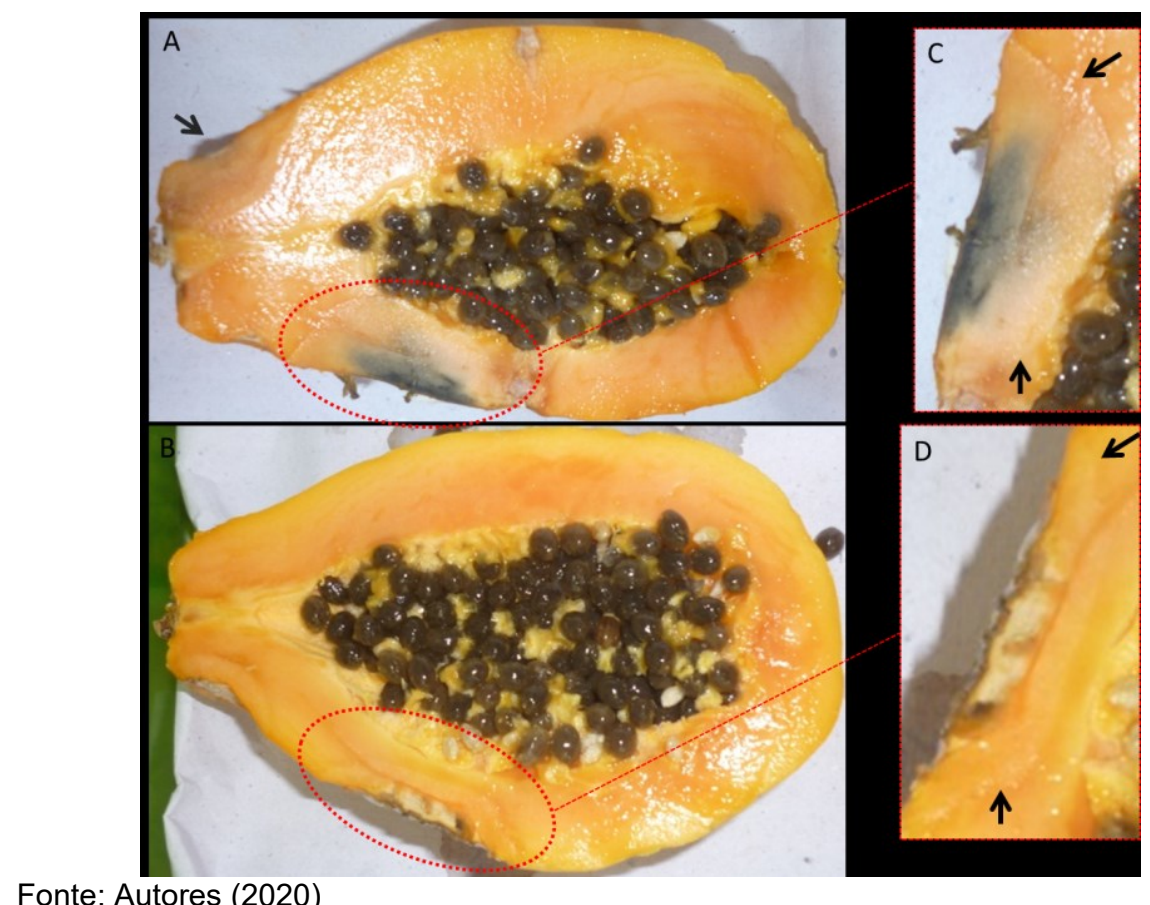

Fonte: Autores (2020)

Aparentemente, o extrato não causa danos naturais na estrutura do fruto, podendo ser aplicado nos mesmos sem prejuízos a comercialização. Trabalhos prévios têm demonstrado a eficácia de outros extratos vegetais na conservação de frutos de mamão evitando o aparecimento de sintomas de antracnose. Siqueira Junior et al. (2012) demonstraram que o tratamento com extratos de mamona (Ricinus cummunis) elimina o aparecimento dos sintomas de antracnose em frutos tratados quando comparados aos frutos não tratados. Mais recentemente, Marinho et al. (2018) descreveram o mesmo padrão de conservação de frutos tratados com extrato de saboeiro (Sapindus saponaria), reforçando a ideia da utilização de extratos vegetais como alternativa para a redução das perdas agrícolas por incidência de antracnose na cultura e consequente melhoria dos padrões 
de produção e qualidade dos frutos cultivados. Serão necessárias mais análises relacionadas a mensuração dos valores nutricionais dos mamões tratados com os extratos visando a aprovação do uso deles no campo.

\section{Conclusões}

O Extrato de $M$. azedarach proporciona a inibição do crescimento micelial dos fungos $F$. oxysporum e $F$. solani aparentemente, promovendo mudanças morfológicas nas hifas. O extrato também reduz a formação dos sintomas da antracnose, provocada pelo fungo $C$. gloeosporioides em frutos de mamão. Apesar da inibição reduzida sobre o crescimento in vitro do fungo, quando usado in natura, o extrato inibe o aparecimento das lesões típicas da doença. Em conjunto, os dados obtidos demonstram a importância do extrato de $M$. azedarach no controle de fungos fitopatogênicos economicamente importantes para o País, caracterizando uma alternativa sustentável de controle de pragas e patógenos, que poderia ser empregada em substituição aos agrotóxicos já utilizados regularmente, e considerados agressivos ao meio ambiente e a saúde humana.

\title{
5 Evaluation of Antifungal Activity of Cinamomo Leaves (Melia Azedarach) on Phytopathogenic Fungi Aiming at an Alternative to Pesticides
}

\begin{abstract}
Bioactive compounds of plant origin have been widely studied because they are natural sources of antifungal substances. In the present work, a hydroethanolic extract was used to extract bioactive compounds from leaves of Melia azedarach plants. Antifungal activity of the M. azedarach extract was then tested against three phytopathogenic fungi: Fusarium solani, Fusarium oxysporum and Colletotrichum gloeosporioides, the latter, well known for causing anthracnose in papaya fruits (Carica papaya L.) in the post-harvest. The hydroethanolic extract exhibited strong inhibitory potential on mycelial growth of $F$. oxysporum, reducing its development by $85 \%$. This inhibition is corroborated by the morphological change caused in fungal mycelia by the addition of $100 \mathrm{ppm}$ of the extract. There was also a $44 \%$ reduction in the growth of $F$. solani, in the presence of the extract. Despite the mild inhibition caused by the extract in the development of the fungus $C$. gloeosporioides (14\%), when applied to fruits, the extract confers a reduction in the development of anthracnose symptoms, maintaining the visual quality of the tested fruits. Results obtained in this work corroborate the idea of using compounds extracted from plants as a sustainable alternative to application of pesticides, promoting the cheapening of the pest and pathogen control process in cultivation, besides constituting a way of controlling less aggressive to environment.
\end{abstract}

Keywords: Anthracnose; Plant extract; Antifungal activity; Papaya fruit.

\section{Referências}

ADEME, A.; AYALEW A.; WOLDETSADIK K. Evaluation of Antifungal Activity of Plant Extracts against Papaya Anthracnose (Colletotrichum gloeosporioides). Journal of Plant Pathology \& Microbiology, v.4, p. 207-210, 2013.

AGROFIT. Sistema de Agrotóxicos Fitossanitário. Consulta de Pragas/doenças e produtos indicados. 2020. Disponível em: http://agrofit.agricultura.gov.br/agrofit_cons/princip al_agrofit_cons. Acesso em: 16 de mar. 2021.

AHMADU, T.; AHMAD, K.; ISMAIL, S.I.; RASHED, O.; ASIB, N.; OMAR, D. Antifungal efficacy of Moringa oleifera leaf and seed extracts against Botrytis cinerea causing gray mold disease of tomato (Solanum lycopersicum L.). Brazilian Journal of Biology, v. 81, p. 1007-1022, 2020.
CAPDEVILLE, G.; JUNIOR, M.T.S.; SANTOS, J.R.P.; MIRANDA, S.P.; CAETANO, A.R.; FALCÃO, R.; GOMES, A.C.M.M. Scanning electron microscopy of the interaction between Cryptococcus magnus and Colletotrichum gloeosporioides on papaya fruit. Pesquisa Agropecuária Brasileira, Brasilia, v.42, p. 1537 1544, 2007.

CHEN, Y.; DAI, G. Antifungal activity of plant extracts against Colletotrichum lagenarium, the causal agent of anthracnose in cucumber. Journal of the Science of Food and Agriculture, v. 92, p.1937-1943, 2012.

GRUJIĆ, S.M.; SAVKOVIĆ, Ž.D.; RISTIĆ, M.S.; DŽAMIĆ, A.M.; LJALJEVIĆ GRBIĆ, M. V.; VUKOJEVIĆ, J.B.; MARIN, P.D. Glandular trichomes, essential oil composition, antiAspergillus and antioxidative activities of Lamium 


\section{REA - Revista de estudos ambientais (Online) \\ v.22, n. 2, p.43-49, jul./dez. 2020}

purpureum L. ethanolic extracts. Archives of Biological Sciences, v. 72, p. 253-263, 2020.

LUNDSTRÖM, N.L.; ZHANG, H.; BRÄNNSTRÖM, A. Pareto-efficient biological pest control enable high efficacy at small costs. Ecological Modelling, v.364, p. 89-97, 2017.

MAPA. Ministério da Agricultura, Pecuária e Abastecimento. MAPA publica extensão de uso de três defensivos agrícolas para pequenas culturas. 2020. Disponivel em: https://www.gov.br/agricultura/pt-

br/assuntos/noticias/mapa-publica-extensao-deuso-de-tres-defensivos-agricolas-para-pequenasculturas. Acesso em: 16 de mar. 2021.

MARINHO, G. J. P.; KLEIN, D. E.; SIQUEIRAJUNIOR, C. L. Evaluation of Soapberry (Sapindus saponaria L.) leaf extract against papaya anthracnose. Summa Phytopathologica, v. 44, p. 127-131. 2018.

MARTINS, J.A.B.; SIQUEIRA-JUNIOR, C.L. Potential control of anthracnose in papaya (Carica papaya) by treatment with plant extracts. Revista Biociência, v. 23, p. 61-68, 2017.

MOHAMED, N.H.; EL-HADIDY, A.M. Studies of biologically active constituents of Verbascum eremobium Murb. and its inducing resistance against some diseases of cucumber. Egyptian Journal of Phytopathology, v.36, p. 33-150, 2008.

NAZ, R.; BANO, A. Phytochemical screening, antioxidants and antimicrobial potential of Lantana camara in different solvents. Asian Pacific Journal of Tropical Disease, Haikou, v. 3, p. 480486, 2013.

ROSADO-ÁLVAREZ, C.; MOLINERO-RUIZ, L.; RODRÍGUEZ-ARCOS, R.; BASALLOTE-UREBA, M.J. Antifungal activity of asparagus extracts against phytopathogenic Fusarium oxysporum. Scientia Horticulturae, v.171, p. 51-57, 2014.

SEEPE, H. A; LODAMA, K. E.; SUTHERLAND, R.; NXUMALO, W.; AMOO, S. O. In vivo antifungal activity of south african medicinal plant extracts against Fusarium pathogens and their phytotoxicity evaluation. Plants, v.9, p. 1668- 1675, 2020.

SEN, A.; BATRA, A. Evaluation of antimicrobial activity of different solvent extracts of medicinal plant: Melia azedarach L. International Journal of Current Pharmaceutical Research, v.4, p. 67-73, 2012.

SILVA, F.A.Z.; AZEVEDO, C.A.V. The Assistat Software Version 7.7 and its use in the analysis of experimental data. African Journal of Agricultural Research, v.11, p. 3733-3740, 2016.

SIQUEIRA-JUNIOR, C.L. Antifungal properties of combined plant extracts against papaya anthracnose. Revista Verde, v. 9, p. 167-172, 2014.

SIQUEIRA JUNIOR, C.L.; FREIRE, M.G.M.; MOREIRA, A.S.N.; MACEDO, M.L.R. Control of papaya fruits anthracnose by essential oil of Ricinus communis. Brazilian Archives of Biology and Technology, v. 55, p.75-80, 2012.

VENTURA, J.A.; COSTA, H.; TATAGIBA, J.S. Manejo das doenças do mamoeiro. In: Martins, D.S.; Costa, A.F.S. (ed.) A cultura do mamoeiro: tecnologias de produção. Editora Incaper, Vitória, Brasil. p. 229-308, 2003.

WANG, Y.; CHEN, C.; ZHAO, X.; WANG, Q.; QIAN, $Y$. Assessing joint toxicity of four organophosphate and carbamate insecticides in common carp (Cyprinus carpio) using acetylcholinesterase activity as an endpoint. Pesticide Biochemistry and Physiology, v. 122, p. 81-85, 2015. 